\title{
BIOMECHANICS MEASUREMENTS IN ARCHERY
}

\section{Zulkifli Ahmad*, Zahari Taha, Hasnun Arif Hassan, Mohd Azrul Hisham, Nasrul Hadi Johari and Kumaran Kadirgama}

\author{
${ }^{1}$ Centre for Sports Engineering, Faculty of Mechanical Engineering, \\ Universiti Malaysia Pahang \\ 26600 Pekan, Pahang, Malaysia \\ Email: "kifli@ump.edu.my \\ Phone: +609-4246286; Fax : +609-4246222
}

\begin{abstract}
The purpose of this study is to measure the biomechanics parameters of the sport of archery and correlate these with the games performance. Archery is becoming a sport that may potentially success in the 2016 Olympic Games. Therefore, research in this sport can be directly relevant to athlete's performance during games tournaments. This research is considered as a preliminary study to measure the biomechanics parameters that can be applied to the professional athlete. Biomechanics parameters such as muscle activity, heartbeat, balance and body posture as well as the draw force line were selected as important parameters related to the athlete's performance. The subject in this study shot arrows on a force plate while a high speed video camera captured the arrow velocity, body posture and elbow angle. Furthermore, these parameters are then correlated with the point of the target or the archer's performance in order to propose the correct shooting technique. The findings of this study are the draw force line angle, the most active muscle, the foot weight balance and the aiming concentration release time. All the results will help both coach and athlete to improve the performance of the sport, and especially assist both beginner level archers aiming to become expert and elite players.
\end{abstract}

Keywords: Biomechanics; sport; archery; muscle; performance.

\section{INTRODUCTION}

Archery is a sport which propel arrows with a bow to the target during shooting (Lee, 2009). Shooting in archery can be summarized as drawing the bow, aiming and releasing the arrow (McKinney \& McKinney, 1997). In archery it is important that the archer is able to hold the pulling force of the bow isometrically at release. Any collapse or movement forward of the draw arm results in a reduction of the velocity of the arrow and this results in the arrow going too slowly towards the target. Therefore, over time improvement and refinement of the bow and arrow are increased to get the highest performance equipment (Mukaiyama, Suzuki, Miyazaki, \& Sawada, 2011). High performance shooting in archery is defined as the ability to shoot an arrow at a given target with accuracy (Soylu, Ertan, \& Korkusuz, 2006). Alignment of the bow arm is important as it will produce a certain angle and if the alignment is incorrect the force applied to the muscles may increase. The forces acting on the archer may vary as the skill and technique levels differ from one athlete to another. It also involves the rhythms of releasing the arrow, which differ between elite and sub-elite archers (Hu \& Tang, 2005). The application of biomechanics in archery is very useful. It helps in the control 
of the movement of muscles and in reducing the fatigue effects that may occur, possibly causing serious injuries in the long term. Moreover, the joint movement is also crucial in analyzing the motion of the human body and to better understand how or why injuries occur (Kristianslund, Krosshaug, \& van den Bogert, 2012). According to the biomechanics principle in archery, the forces acting on the bones should be maximized while the force acting on the muscles should be minimized in order to reduce the injury impact to the archer. The force applied should be more on the bones than on the muscles because the bones do not get tired while muscles most certainly become fatigued.

The angle and position of the elbow play an important role in subjecting force to the shoulder. Therefore, the position of the elbow should be aligned with the line of force, as the distance of the shoulder line and line of force at the shortest. This line is called the draw force line (DFL). On the other hand, an incorrect position of the elbow causes muscle action to maintain the draw force line level. If this happens continuously, it may cause muscle fatigue, and subsequently impair the performance. In order to minimize the lateral forces of the arm, the angle between the forearm and the DFL should be small. During archery shooting, the upper limb muscles are more active than the lower-limb muscles due to the need to pull and hold the bow until the arrow is released. However, extremely vigorous muscle needs more strength to pull and hold the bow in the forearm. The forearm muscles which are commonly involved in this shooting are the flexor digitorum and extensor digitorum. Also involved are the deltoid muscle, trapezius muscles, medial epicondylitis and pectoralis major muscle. By using electromyography (EMG) equipment, the muscle activity can be detected and analyzed. One of the key elements to obtain better stability in archery is to have a good stance and posture. The term stance specifically refers to the standing posture of the archer. Stance requires strength in the legs, and the right stance can help to maintain stability while standing for an extended period. Therefore, the release phase must be well-balanced and highly reproducible to achieve commendable results in an archery competition (Tinazci, 2011). The archer is required to stand still for quite a period of time and having the correct stance help shim to maintain his body balance for the whole of the arrow shooting process. A good stance can help the archer to sustain his stability for a longer time and hence helps to aim better at the target board. When the body is stable, the archer's shots will be more consistent and less frustrating. The different levels achieved by archers represent different styles of stance and different weight distribution, which depend on the body posture, height and bone structure.

General biofeedback is a tool to provide human beings with enhanced awareness of their mind-body lineage, increased control over their physiology, and increased access to self-regulation strategies (Donald \& Sue Vietta, 2012). Biofeedback can awaken human creativity and enable individuals to reach higher states of consciousness. The biofeedback device will help to show the archer's concentration. Therefore, the higher the frequency level of an athlete, the greater the athlete's concentration on the target. The purpose of this study is to measure the biomechanics parameters involved in the archers' performance. These measurements are considered as a preliminary study in order to optimize the equipment, location and subject constraints. In conducting this experiment, there are several hypotheses to test involving increasing the performance by ensuring that the draw force is in line, reducing the muscle activity, weight balancing and aiming concentration. 


\section{METHODOLOGY}

An experienced coach was selected as a subject to shoot the arrow on the force plate indoors. The archer was right-handed and a recurve bow was used to shoot the arrow. The subject's physical characteristics are 23 years of age, height $180 \mathrm{~cm}$ and body weight $84 \mathrm{~kg}$. An EPIX high-speed camera, Shimmer electromyography (EMG) module, and AMTI force plate and biofeedback device were used to setup the experiment. Since the measurement experiments were being run simultaneously, some of the equipment was installed on the subject's body, namely the EMG and biofeedback device.

\section{EPIX High-Speed Camera}

The high-speed camera was used to ensure that the subject was in the correct position for the draw force line. The correct technique prevents the injury called "rotor cuff", which is very painful and requires a long time to repair. Figure 1 shows the position of the two high-speed cameras required to record the top view and the sagittal view of the athlete, when drawing the bow, aiming and releasing. The two cameras were synchronized and set to 150 frames/s, as shown in Figure 2. In order to measure the draw force line, 12 reflective markers were placed on the subject's wrists, elbows and shoulders. Then, XCAP software was used to measure the angle, distance and draw force line accurately.
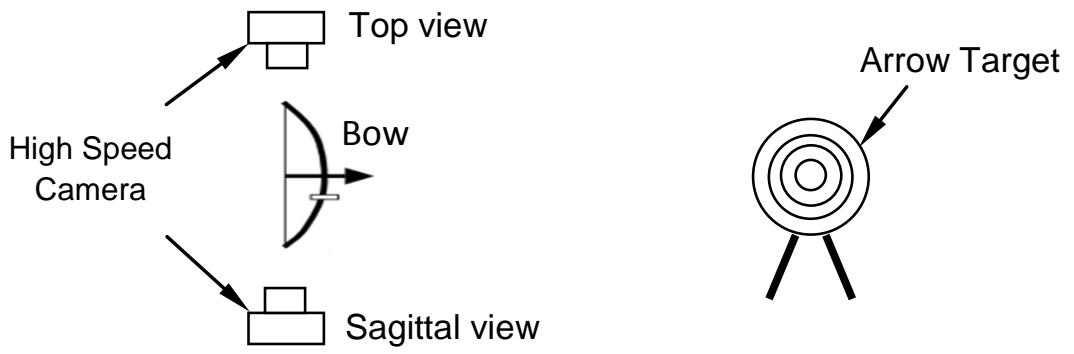

Figure 1. High-speed camera set up.

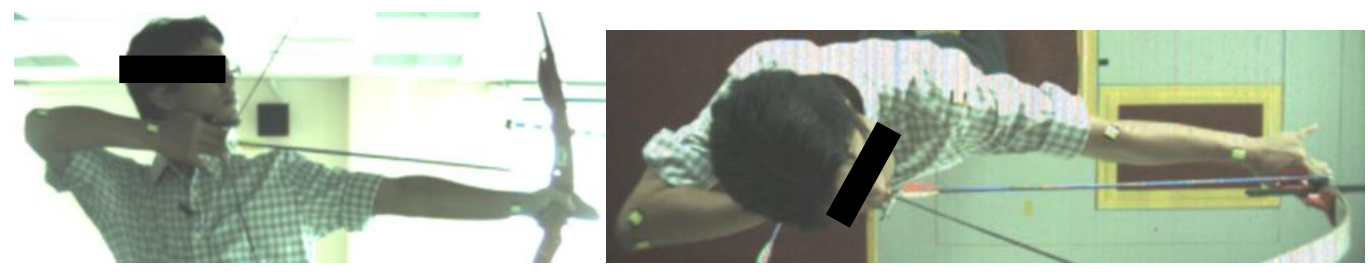

Figure 2. Camera synchronization for lateral view (left) and top view (right)

\section{Shimmer Electromyography}

Electromyography (EMG) is a technique for analyzing muscle activity by evaluating and recording the electrical activity produced by those muscles. During archery shooting, the muscles involved are the deltoid, flexor digitorum and extensor digitorum. However, only the right deltoid and flexor digitorum were selected for observation because most of the muscle activity takes place there. The muscle activation was 
recorded at a sampling frequency of $1000 \mathrm{~Hz}$. All the signals were recorded for the three stages of drawing, aiming and releasing. Then the results obtained were plotted in the graph. Figure 3 indicates the EMG measurement locations on the subject.

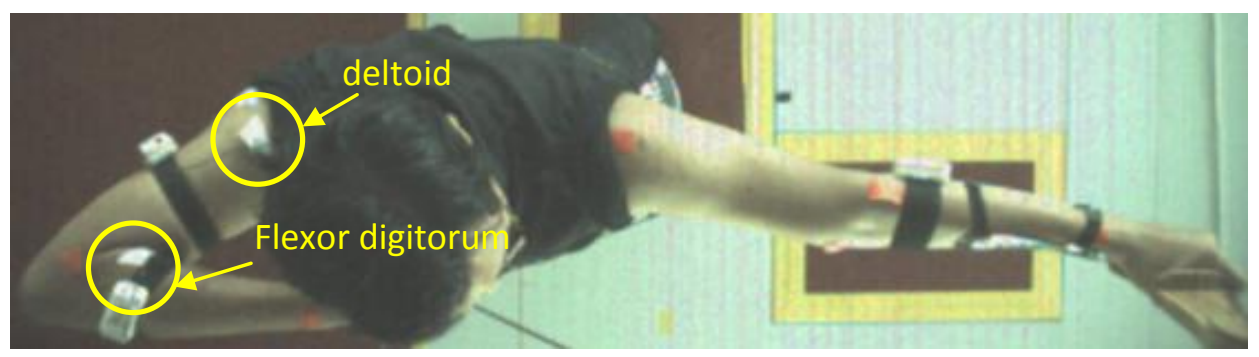

Figure 3. EMG measurement locations

\section{AMTI Force Plate}

Archery can be described as a static sport, which involves a stable sequence of performed movement patterns (Hayri \& Rafet, 2010). The AMTI force plates were used to acquire the signal of the force acting on the feet, which is called the ground reaction force (GRF). During this experiment, the subject took up his position on the platform with his left foot placed on the force plate. Data was collected for five seconds, but only the data from the first four seconds of the experiment were used, this being the time period the shooting attempts actually required, the stages of which are drawing, aiming and releasing the arrow. The subject's weight and height were measured before starting the experiment. After completion of the shooting process, the force data can be analyzed using BioAnalysis software to obtain the weight distribution on the foot for each millisecond.

\section{Biofeedback Device}

The biofeedback device is fitted as shown in Figure 4 below to obtain the frequency level of the archer. The device should be calibrated first for about 60 seconds in order to receive the signals. After the equipment is setup, the archer starts to shoot. The archer releases the arrow after aiming for three seconds, counted by a stopwatch. The results of the shot points will be viewed from the digital camera. For biofeedback analysis, the BioGraph Infiniti Physiology Suite software was used to record and analyze the data. The blood volume pulse (BVP) sensor was attached on the archer's finger as shown in Figure 4.

The BVP sensor does not require skin preparation as it is placed directly in contact with the skin. The device takes the data on the archer's BVP by shining infrared light through the finger and measuring the amount of light reflected by the skin. The amount of reflected light varies during each heart beat as more or less blood rushes through the capillaries. The sensor converts the reflected light into an electrical signal that is processed using biofeedback software. 


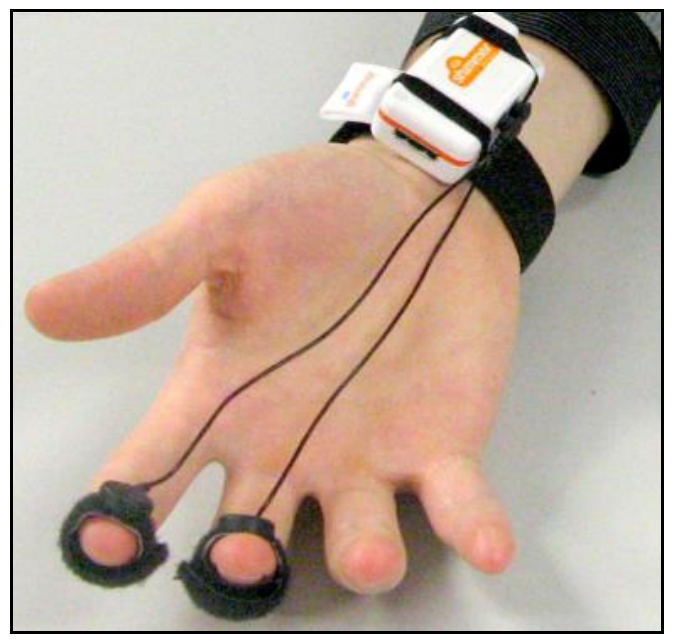

Figure 4. The BVP sensor attached to the finger

\section{RESULTS AND DISCUSSION}

The findings in this study were divided into four sections as described in the methodology. These are the draw force line, muscle activity, weight balancing distribution and aiming concentration during shooting. This section presents the biomechanics parameters that were obtained from the experiment.

\section{Draw Force Line}

The point of origin is at the elbow, which acts as a reference point to determine the distance and angle from the elbow to the shoulder. As shown in Figure 5, the angle was measured as $45^{\circ}$ clockwise. A line was drawn between each joint and marked by a yellow marker. The elbow is not in line, which is common for a recurve archer. However, this angle size is still considered acceptable given the small distance from the dotted arrow DFL.
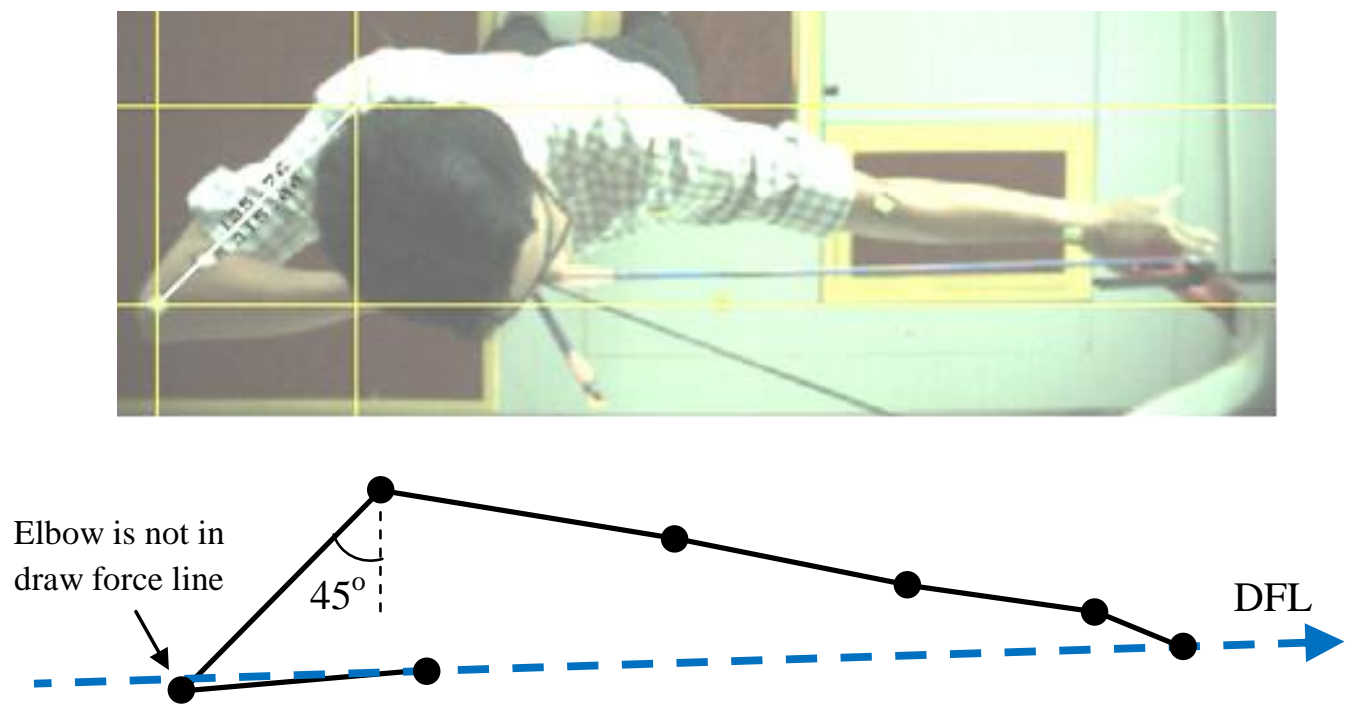

Figure 5.DFL measured from top view 
A similar measurement also occurred with the DFL from the lateral view. The elbow is still not in line, which requires the drawing arm's biceps and triceps to take all the force and so creates fatigue. Ideally, the elbow should be within the DFL in order to reduce the muscle fatigue as well as to prevent injury. Repetition of the same technique will affect the performance of the archer. The results shown in Figure 6 illustrate that the DFL is almost straight, with just a slight angle out. This is in fact common with recurve bow archers. Therefore, we can assume that this is an experienced and knowledgeable archer.

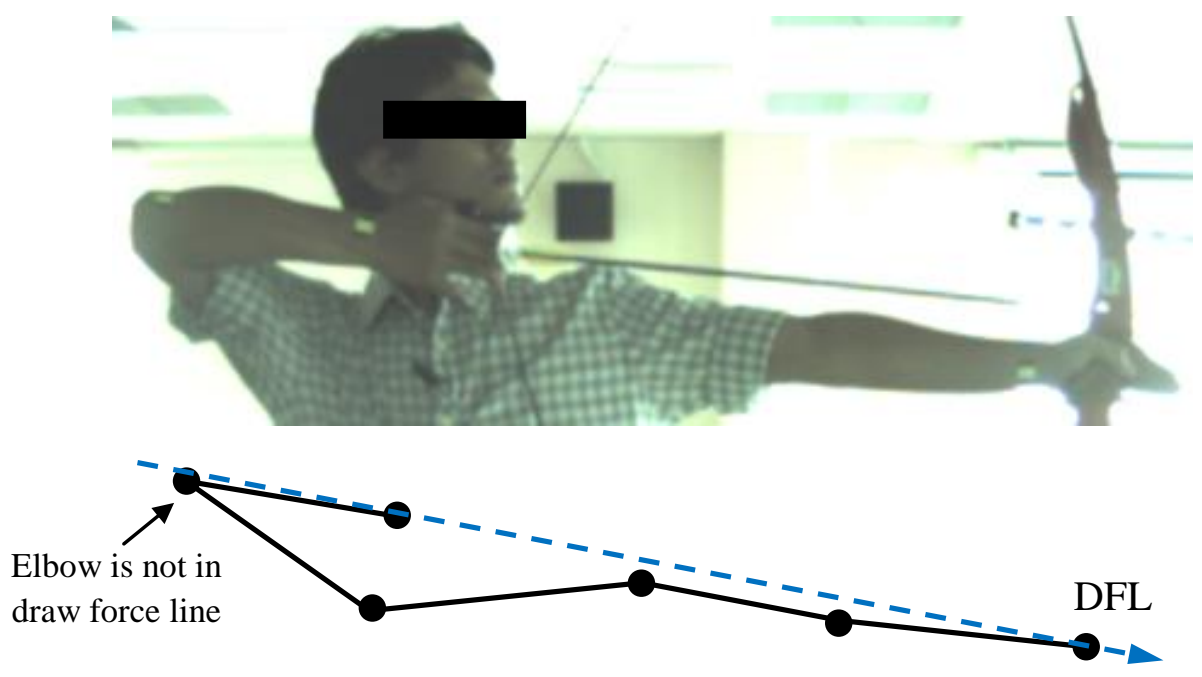

Figure 6.DFL measured from the lateral view

\section{Muscle Activity}

For this section, the measurement obtained from this equipment is the signal from the muscle activated during the shooting process. The muscles considered for this study are only in the archer's right arm, namely the flexor digitorum and deltoid muscles. Both of these muscles can cause an injury to the archer and these were the main concern (Ertan, Kentel, Tümer, \& Korkusuz, 2003; Soylu, et al., 2006; Tinazci, 2011). Figure 7 indicates the activity of the flexor digitorum muscle during shooting. There are three phases involved in this result; setting the bow, aiming and releasing the arrow. These phases are represented by the symbols $\mathrm{A}, \mathrm{B}$ and $\mathrm{C}$ respectively. The first phase, A, represents setting the bow and this pre-draw phase has a low amplitude compared to the $\mathrm{B}$ phase. This is because in this phase the muscle is not fully active when pulling the string, and still has to put more force on it.

The highest amplitude in this graph, $\mathrm{f}$ more than $2.5 \mathrm{mv}$,occurs between 0.7 and 1.4 seconds. This represents the B phase which is the full draw and aiming phase. It has the highest amplitude because, at that time, the right arm muscle is at its most active in holding the string for a few seconds, which requires more strength. At 1.6 seconds, the activity increased and then decreased abruptly in phase $\mathrm{C}$ with the release of the arrow. This produces some knocking on the arm as well as the muscle. The deltoid muscle pattern is shown in Figure 8. Although the pattern is very similar, the values of muscle activity are different. The difference is in phases B and C. This illustrates that the activity of the deltoid muscle is higher than the flexor digitorum due to the location of 
the muscle. Hence, the deltoid muscle in the shoulder joint is more crucial than the flexor digitorum because the drawing arm force depends on the strength of that muscle.

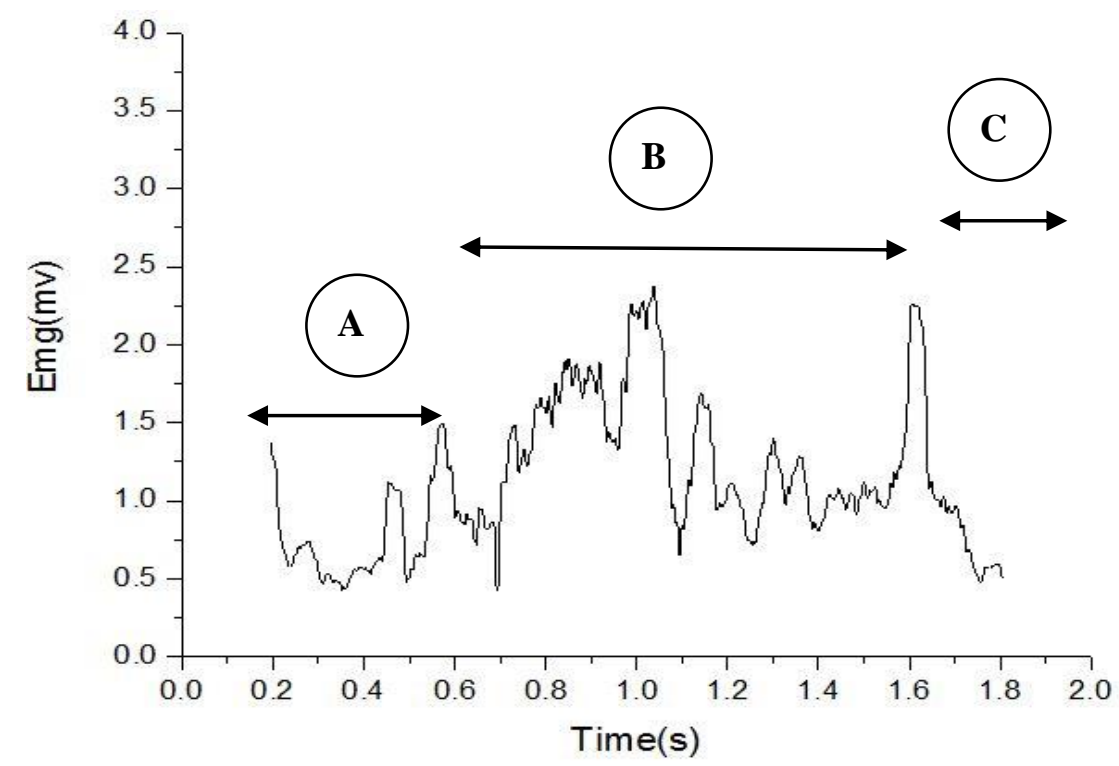

Figure 7. Flexor digitorum muscle

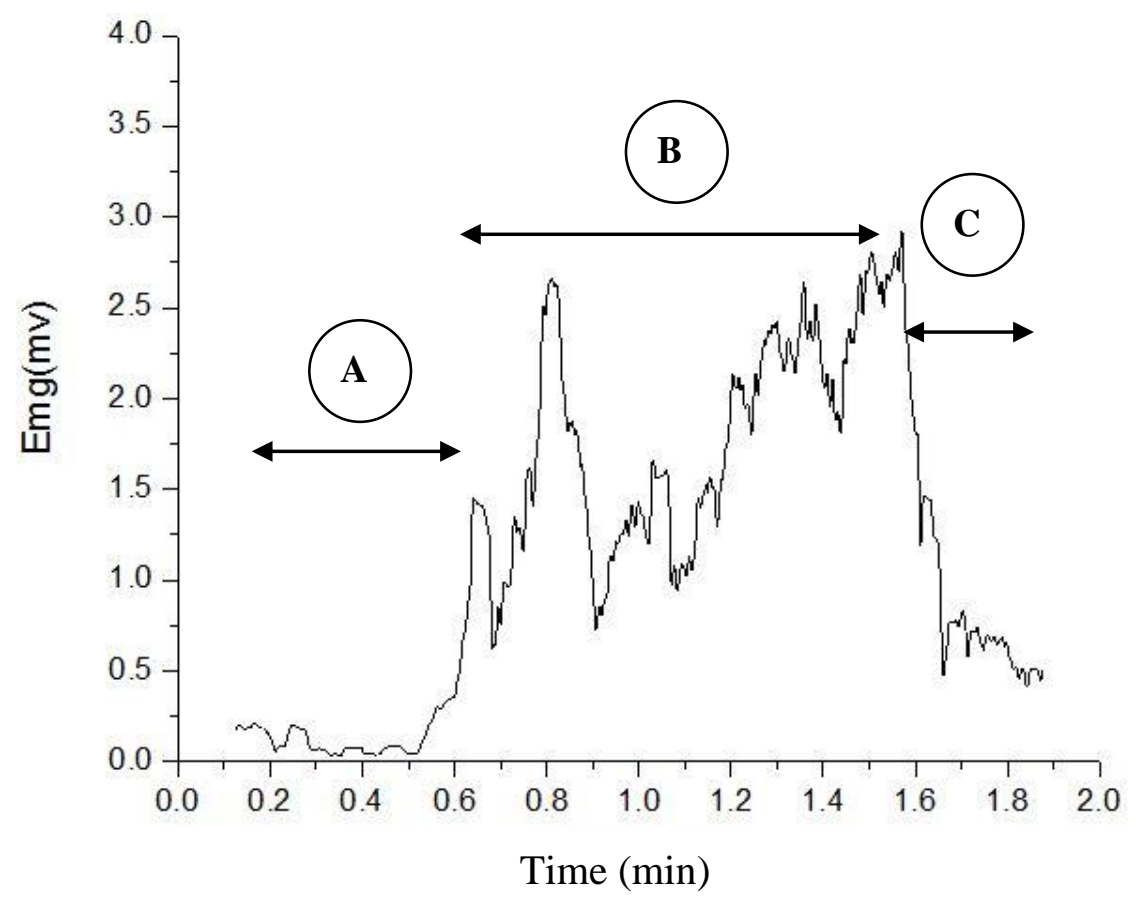

Figure 8.Deltoid muscle

\section{Weight Balancing Distribution}

To get good results in an archery competition requires a well-balanced and highly reproducible release during the shooting. However, only the left foot was measured by the force plate because the plate is small compared to the archer's stance distance. The total weight of the subject is about $84 \mathrm{~kg}$. To ensure a good balance, the total weight 
was halved into $42 \mathrm{~kg}$. Since the weight relates to gravity, the z-axis value was selected as observation data to analyze. The maximum weight on the force plate is $44.12 \mathrm{~kg}$, as plotted in Figure 9.

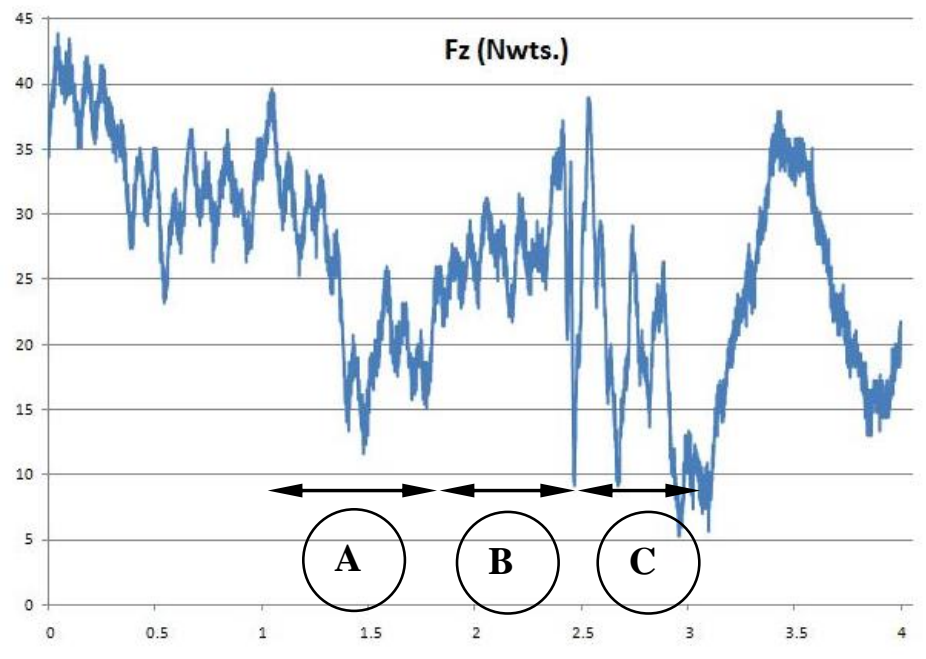

Figure 9. Weight distribution in z-axis

The data was divided into three stages, namely drawing, aiming and releasing. The drawing stage was from 0 to 1.2 seconds, aiming was from 1.2 to 2.8 seconds, and releasing was from 2.8 to 4 seconds. During the drawing stage, the body balance is not consistent, as is also the case in the aiming stage, when it is affected by the bow and the need to create the moment for the subject's stance. This shows that in the shooting process, the weight distribution is more on the right foot than the left foot. In the aiming stage, the archer tries to stabilize his weight distribution along time. However, just after the arrow is released, the weight acting on the left foot is sharply reduced for a brief instant. The weight change indicates that the weight distribution acting on the archer's left foot is disturbed upon the release of the arrow.

\section{Aiming Concentration}

The results here present the measurement of aiming concentration for a three second period. The BVP value for each frequency is shown in Table 1. The mean total heart rate for three attempts is $112.10 \mathrm{bpm}$. Physically, this is higher than the normal heart rate which is $90 \mathrm{bpm}$. In this condition, the subject is under pressure but is still able to do the shooting.

Table 2shows the frequency level of the archer during the aiming phase. The aiming concentration depends on the higher frequency (HF) and can be related to the performance results. The higher the frequency level, the better the concentration of the archer. Three attempts were made with the same aiming time. The results were counted from the target board for each attempt. During the first attempt, the frequency level of the archer was quite high and the arrow hit the yellow area. In the second trial, the frequency level was lower than before and the arrow hit the red area, which is quite far from the center. In the last trial, the frequency level increased again and the arrow struck close to the yellow area. This shows that the frequency level is directly related with the subject's performance in terms of the concentration factor. 
Table 1. BVP value for aiming

\begin{tabular}{lc}
\hline Description & Value \\
\hline B: BVP HR mean (beats/min) & 112.10 \\
B: BVP VLF \% power mean & 16.61 \\
B: BVP LF \% power mean & 36.51 \\
B: BVP HF \% power mean & 46.88 \\
B: BVP VLF total power mean & 1216.70 \\
B: BVP LF Total power mean & 3183.65 \\
B: BVP HF total power mean & 3980.56 \\
B: BVP LF/HF (means) & 0.80 \\
\hline
\end{tabular}

Table 2. Frequency value with shot points results

\begin{tabular}{ccccc}
\hline Attempts & $\begin{array}{c}\text { Very Low } \\
\text { Frequency (VLF) }\end{array}$ & $\begin{array}{c}\text { Low Frequency } \\
(\text { LF })\end{array}$ & $\begin{array}{c}\text { Higher } \\
\text { Frequency (HF) }\end{array}$ & Results \\
\hline 1 & 22.34 & 30.41 & 47.83 & 8 \\
2 & 8.21 & 52.31 & 40.19 & 7 \\
3 & 17.92 & 31.57 & 50.51 & 8 \\
\hline
\end{tabular}

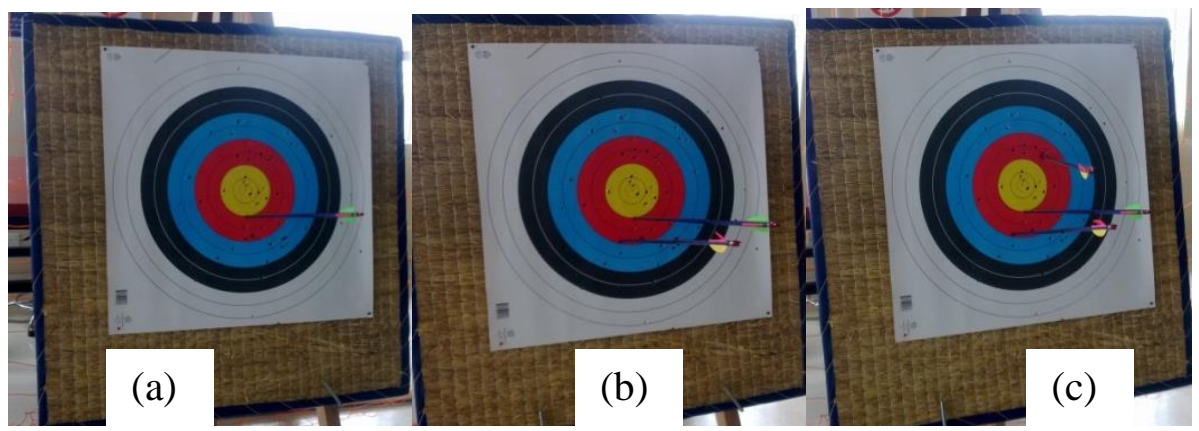

Figure 10.(a) $1^{\text {st }}$ attempt, (b) $2^{\text {nd }}$ attempt, and (c) $3^{\text {rd }}$ attempt

\section{CONCLUSIONS}

In conclusion, the biomechanics parameters selected in this study clearly affected the archer's performance. The wrong drawing technique will create muscle fatigue due to increasing the muscle activity. Furthermore, it will also cause injury to the archer if continuously repeated. In order to get a good result, stabilization of the weight balance is crucial. It reduces the vibration of the body and the equipment itself before shooting the arrow. Aiming concentration has a direct relationship with the archer's frequency level. Consequently, it was confirmed that all the biomechanics parameters discussed in this study were associated with the archer's performance. For future study, athletes with different levels of experience will be the subjects: beginner, intermediate and elite. Therefore, beginners will be trained to become as good as elite players in a short time. 


\section{ACKNOWLEDGEMENTS}

The authors would like to thank the Universiti Malaysia Pahang and Ministry of Higher Education for the financial support under grant RDU110702; also the experienced coach Mr. Nurulakla for his time helping us to perform the right shooting technique, and finally the students in the CenSE group (archery) who helped to complete this experiment.

\section{REFERENCES}

Donald, M., \& Sue Vietta, W. (2012). The use of General Biofeedback in the Pursuit of Optimal Performance: Wiley-Blackwell.

Ertan, H., Kentel, B., Tümer, S. T., \& Korkusuz, F. (2003). Activation patterns in forearm muscles during archery shooting. Human Movement Science, 22(1), 3745.

Hayri, E., \& Rafet, I. (2010). Archery biomechanics: a kinematical approach. . Paper presented at the Annual Meeting of the American Society of Biomechanics.

Hu, C. M., \& Tang, W. T. (2005). Muscle activation by Olympic female archers at different releasing rhythms. Paper presented at the 20th International Society of Biomechanics Congress, Cleveland, Ohio.

Kristianslund, E., Krosshaug, T., \& van den Bogert, A. J. (2012). Effect of low pass filtering on joint moments from inverse dynamics: Implications for injury prevention. Journal of Biomechanics, 45(4), 666-671.

Lee, K. H. (2009). Evaluation of Attention and Relaxation Levels of Archers in Shooting Process.

McKinney, W., \& McKinney, M. (1997). Archery. London: Brown \& Benchmark.

Mukaiyama, K., Suzuki, K., Miyazaki, T., \& Sawada, H. (2011). Aerodynamic properties of an arrow: Influence of point shape on the boundary layer transition. Procedia Engineering, 13, 265-270.

Soylu, A. R., Ertan, H., \& Korkusuz, F. (2006). Archery performance level and repeatability of event-related EMG. Human Movement Science, 25(6), 767-774.

Tinazci, C. (2011). Shooting dynamics in archery: A multidimensional analysis from drawing to releasing in male archers. Procedia Engineering, 13, 290-296. 